\title{
Tumor Targeting
}

\author{
Dario Neri*
}

\begin{abstract}
Cancer chemotherapy relies on the expectation that anti-cancer drugs will preferentially kill rapidly dividing tumor cells, rather than normal cells. Since a large portion of the tumor cells has to be killed in order to obtain and maintain a complete remission, large doses of drugs are typically used, with significant toxicity towards proliferating non-malignant cells. Our research focuses on the targeted delivery of therapeutic agents to the tumor environment by means of specific ligands (antibodies or small organic binding molecules) to tumor-associated antigens. In most cases, we target accessible antigens (such as the EDB domain of fibronectin or the $\mathrm{C}$ domain of tenascin-C) which are abundantly expressed around tumor blood vessels, but virtually undetectable in normal tissues.
\end{abstract}

Keywords: Angiogenesis · Antibody engineering · Encoded self-assembling chemical libraries · Human antibodies · Tumor targeting

\section{Tumor Targeting and Vascular Targeting}

The majority of pharmacological approaches for the treatment of solid tumors suffers from poor selectivity, thus limiting dose escalation (i.e. the doses of drug which are required to kill tumor cells cause unacceptable toxicities to normal tissues).

One avenue towards the development of more selective, better anti-cancer drugs consists in the targeted delivery of bioactive molecules (drugs, cytokines, procoagulant factors, photosensitizers, radionuclides, etc.) to the tumor environment by means of binding molecules specific for tumor-associated antigens.

Our laboratory uses human antibody derivatives for the selective targeting of solid tumors. In most cases, we use antibodies specific to components of the modified extracellular matrix with a vascular pattern of expression, as these antigens are abundant,

${ }^{*}$ Correspondence: Prof. Dr. D. Neri

Swiss Federal Institute of Technology Zurich Institute of Pharmaceutical Sciences

Department of Chemistry and Applied Biosciences Wolfgang-Pauli-Str. 10

ETH Hönggerberg

$\mathrm{CH}-8093$ Zürich

Tel.: +41446337428

Fax: + 41446331301

E-Mail: neri@pharma.ethz.ch stable and readily accessible for biopharmaceuticals injected intravenously (Fig. 1).

\section{Proteomic Approaches for the Discovery of Vascular Targets}

A number of tumor-associated antigens (such as the EDB domain or fibronectin [1], the $\mathrm{C}$ domain of tenascin- $\mathrm{C}$ [2] and the prostate-specific membrane antigen [3]) display a vascular pattern of expression in solid tumors, thus allowing the selective delivery of antibody-derivatives to the tumor neo-vasculature. The promising tumor targeting experiments observed with monoclonal antibody derivatives specific to these antigens, in animal models and in patients with cancer, have motivated us to develop methodologies for the discovery of novel tumor-associated vascular targets.

Our laboratory biotinylates proteins intimately associated to vascular structures by terminal perfusion of tumor-bearing mice with reactive ester derivatives of biotin (e.g. sulfo-NHS-LC-biotin; Pierce) [4]. This procedure washes away from circulation both cells and proteins which could

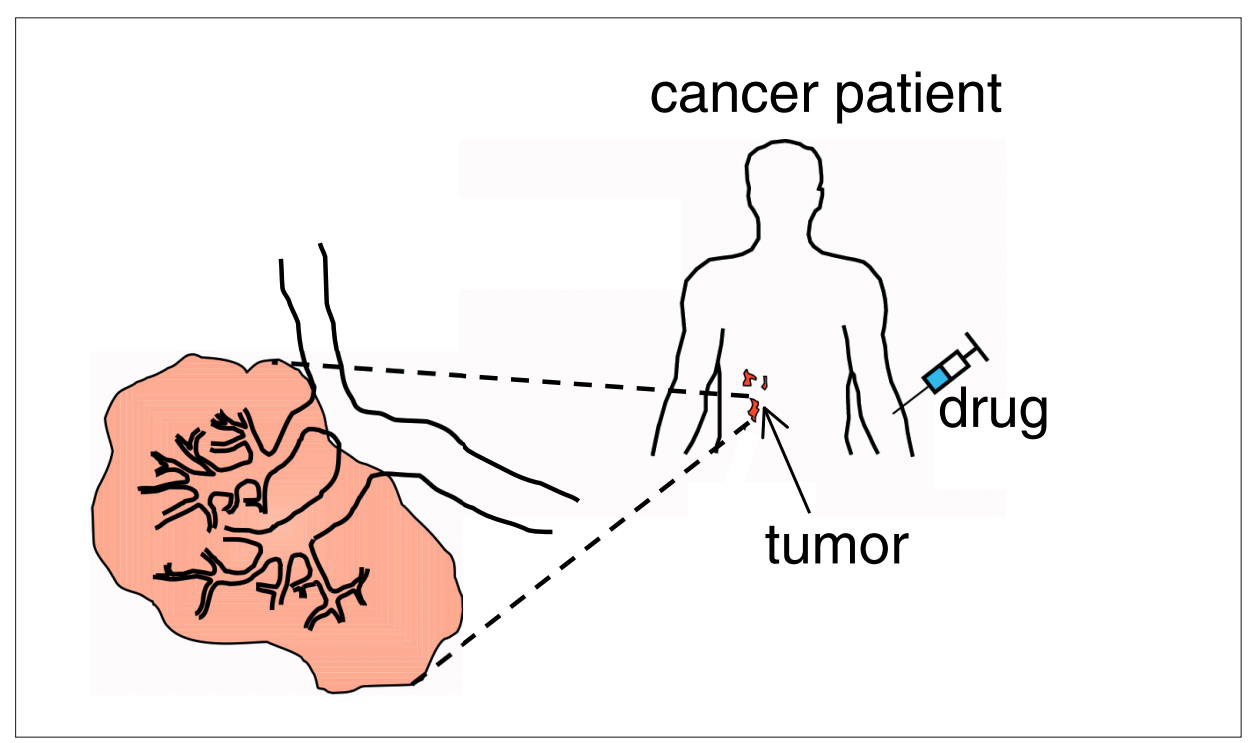

Fig. 1. Schematic representation of tumor targeting. An agent, injected intravenously, preferentially localizes to tumors by virtue of its specific binding to a tumor-associated antigen. Tumor markers located on tumor blood vessels are attractive for tumor targeting, because of their accessibility and of the therapeutic options which become available (e.g. promoting the infarction of solid tumors). 
compete with the biotinylation reaction. As a result, accessible proteins (e.g. membrane proteins, extracellular matrix components) carrying primary amino groups (e.g. unprotected exposed N-termini or lysine sidechains) are covalently modified with biotin. After capture of biotinylated proteins on streptavidin resin, the comparative proteomic analysis of biotinylated proteins in different organs and tumors is performed using either gel-electrophoretic or gel-free methodologies, relying on mass spectrometry for the identification and relative quantification of protein-derived peptides (Fig. 2).

Recently, the group of Jan E. Schnitzer has reported the use of colloidal silica for the in vivo coating of the vasculature by terminal perfusion of tumor-bearing rats. The physical modification enabled the enrichment of silica-coated structures such as luminal cell plasma membranes and their caveolae, followed by the proteomic investigation of the purified endothelial cell pro- teins. This analysis led to the identification of Annexin A1 as a promising novel marker of tumor angiogenesis, and to the demonstration that monoclonal antibodies to this antigen efficiently localize to tumors in a rat lung metastasis model [5].

\section{Human Monoclonal Antibodies}

At present, antibodies are the only general class of affinity reagents that can be generated rapidly and with high binding affinity and specificity against virtually any molecular target. As a consequence, monoclonal antibodies are used in a broad range of applications, from basic biomedical research to clinical analytics, from catalysis to biopharmaceuticals. Several antibodies have recently been approved in the U.S. and in Europe for therapeutic applications.

Traditionally, monoclonal antibodies have been produced by hybridoma technology, following the immunization of mice or

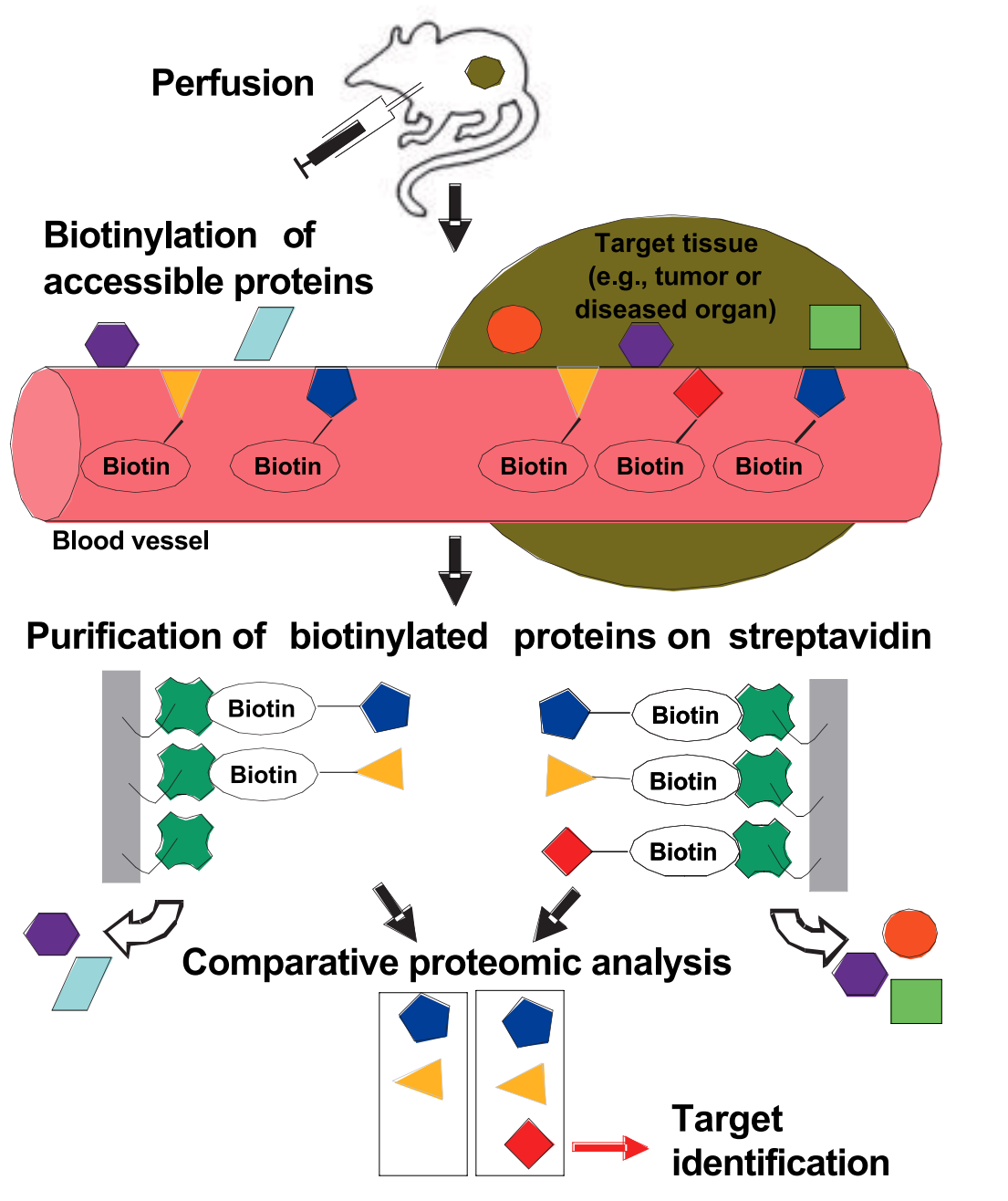

Fig. 2. Schematic representation of the in vivo biotinylation procedure for the comparative proteomic analysis of vascular proteins in different organs and in tumors. Tumor-bearing animals, under terminal anesthesia, are perfused with a solution containing a reactive ester derivative of biotin. Biotinylated proteins in different tissue specimens are then purified on streptavidin-coated resin and submitted to a comparative proteomic analysis. rats. As rodent monoclonal antibodies are immunogenic in patients, the trend in the pharmaceutical industry is to develop humanized or fully human antibodies.

Antibody phage technology, pioneered by the group of Sir Greg Winter [6], has revolutionized the way human antibodies are generated. When pure antigen is available, good quality human antibodies can be isolated in less than a week. The technology is based on the surface display of recombinant antibody fragments (e.g. scFv fragments) on filamentous phage, as fusion proteins to the minor coat protein pIII. The resulting phage particle couples the binding property of the antibody on the surface with the gene (located inside the phage particle) coding for the antibody fragment (Fig. 3A). Typically, libraries containing several billion human antibodies are panned with an antigen immobilized on a solid support, yielding monoclonal antibody fragments, which can be expressed in bacteria or other organisms.

In our laboratory, a synthetic human antibody library has been cloned [7], which contains 4 billion antibody clones and which has yielded a number of useful binding specificities, including antibodies to the EDB domain of fibronectin, a marker of angiogenesis [8], which have been shown to efficiently target solid tumors in animal models and in patients with cancer (Fig. 3B).

\section{Antibody Derivatives for Tumor Targeting Applications}

Angiogenesis, i.e. the proliferation of new blood vessels from pre-existing ones, is an underlying process in many human diseases, including cancer, blinding ocular disorders and rheumatoid arthritis. The ability to selectively target and occlude neovasculature is potentially useful in diagnosis and treatment of angiogenesis-related diseases.

A good-quality marker for both tumoural and non-tumoural neovasculature is the extra-domain B (EDB) of fibronectin, a sequence of 91 aminoacids that can be inserted into the fibronectin molecule by a mechanism of alternative splicing.

To date, the production of monoclonal antibodies directly recognizing the EDB domain has not been possible using hybridoma technology, because of tolerance. In collaboration with Prof. Dr. Luciano Zardi (Istituto Nazionale per la Ricerca sul Cancro, Genova, Italy), we have overcome this problem using large synthetic antibody phage libraries, generating several highaffinity antibody fragments specific for fibronectin containing the EDB domain [9], and mapping their epitope onto the threedimensional structure of the antigen [10]. 

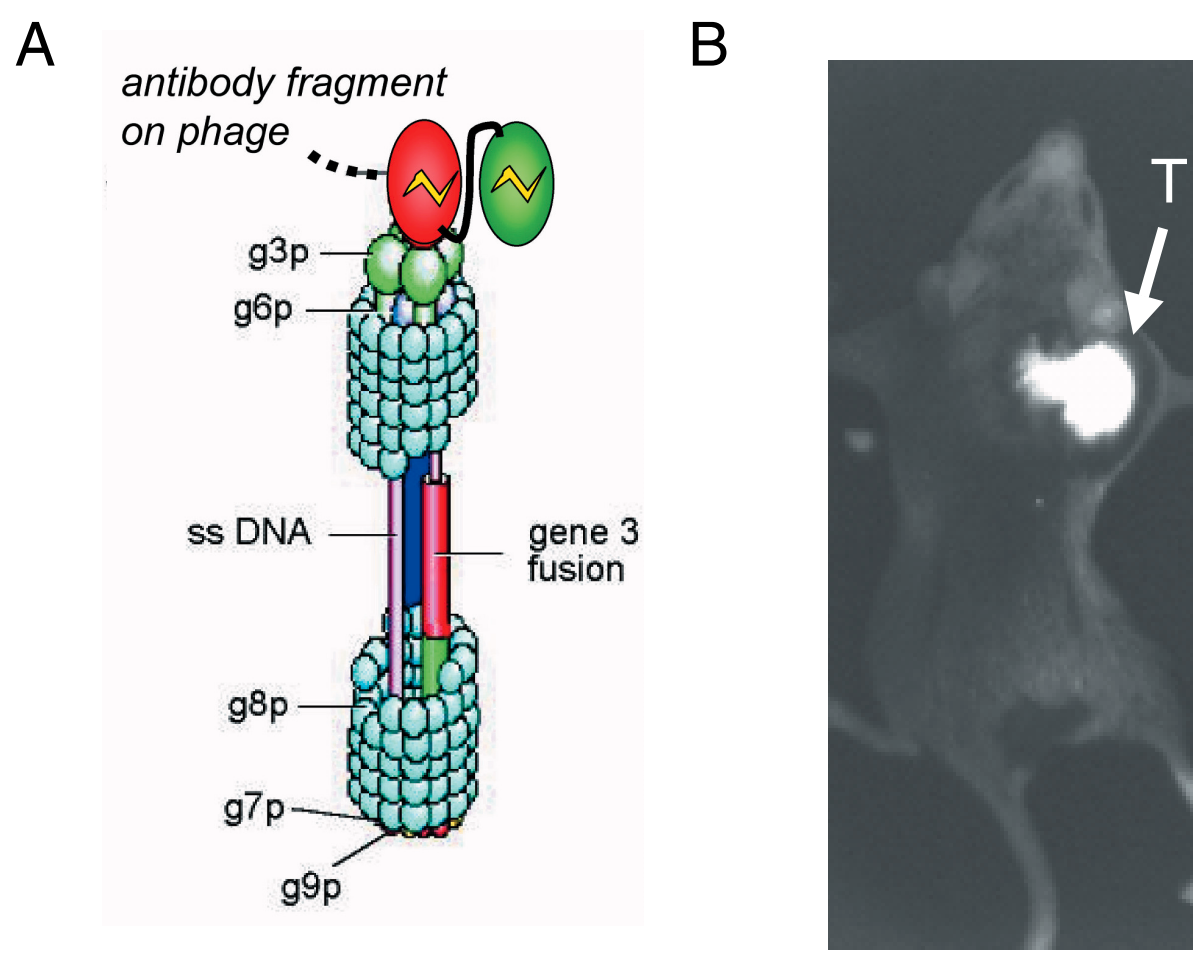

Fig. 3. (A) Schematic representation of antibody phage display. Small fragments of human antibodies (typically in scfv format) are displayed on the surface of filamentous phage, as fusion proteins to the minor coat protein plll. The resulting phage particle couples the binding property of the antibody on the surface with the gene (located inside the phage particle) coding for the antibody fragment. Typically, libraries containing several billion human antibodies are panned with an antigen immobilized on a solid support, yielding monoclonal antibody fragments which can be expressed in bacteria or other organisms. (B) Immunophotodetection of an F9 teratocarcinoma (' $T$ ') in an immunocompetent Sv129 mouse, $24 \mathrm{~h}$ after the injection of the miniantibody SIP(L19) labeled with the infrared fluorophore Cy7.

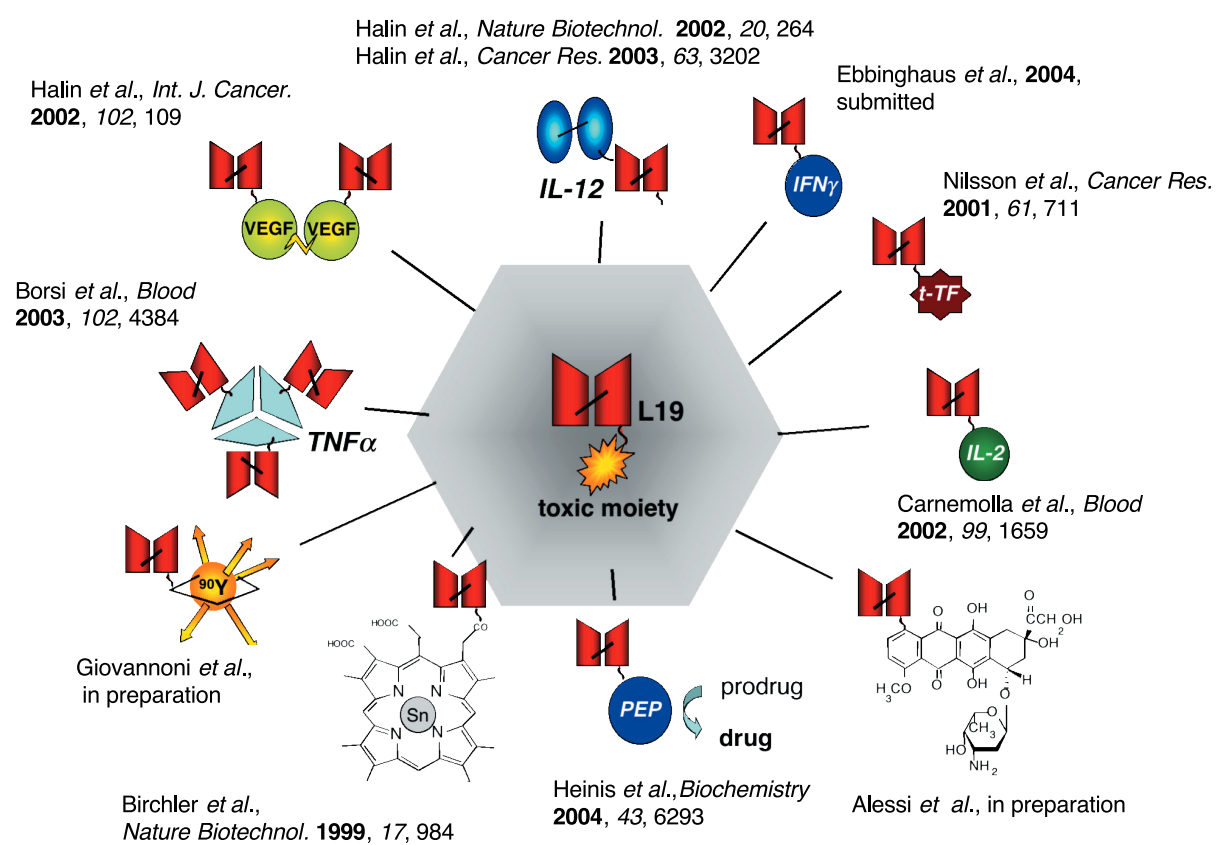

Fig. 4. Schematic representation of therapeutic antibody derivatives, based on scFv(L19), a human antibody fragment specific to the EDB domain of fibronectin. References to the articles describing the performance of these antibody derivatives are indicated.
These antibodies stain vascular structures in tumour sections and selectively target tumour neovasculature, as shown in tumourbearing mice using infrared fluorescence and radioactive techniques. Increased binding affinity leads to improved targeting of tumoural angiogenesis, as demonstrated by biodistributions studies performed using the L19 antibody fragment with affinity for the ED-B domain in the picomolar range and L19 mutants with reduced affinity [11][12].

The ability of radiolabeled L19 to target tumors in patients with cancer has recently been demonstrated using scintigraphic detection methods [13]. A number of derivatives of the L19 antibody (fusions to cytokines, pro-coagulant factors, photosensitizers, drugs, radionuclides, etc.) have been studied in animal models (Fig. 4; see references therein). The results obtained in syngeneic, immunocompetent animal models have been impressive, particularly for the antibody-cytokine fusions, which were efficacious in aggressive rodent models of cancer which are not cured by chemotherapy. These observations are likely to be of therapeutic relevance, since the ED-B domain of fibronectin, a naturally-occurring marker of angiogenesis identical in mouse and man, is expressed in the majority of aggressive solid tumors, but is undetectable in normal vessels and tissues.

\section{By-passing Antibodies: Encoded Self-assembling Chemical Libraries}

For future pharmaceutical developments, it would be attractive to replace antibodies with small organic binding molecules, which retain the high-affinity binding to the target antigen of choice and a selective in vivo localization at sites of disease.

The isolation of small organic molecules capable of specific binding to biological targets is a central problem in chemistry, biology and pharmaceutical sciences. The specific binding to the biological target is not per se sufficient to turn a binding molecule into a drug, as it is widely recognized that other molecular properties (such as pharmacokinetic behavior and stability) contribute to the performance of a drug. However, the isolation of specific binders against a relevant biological target typically represents the starting point in the process, which leads to a new drug.

We have invented and patented a technology (termed 'ESACHEL'; Encoded self-assembling chemical libraries), which may allow the rapid identification of highaffinity organic binding molecules, capable of specific recognition to biological targets (e.g. proteins). In ESACHEL, we use selfassembling libraries of organic molecules (forming dimers or trimers), where each or- 


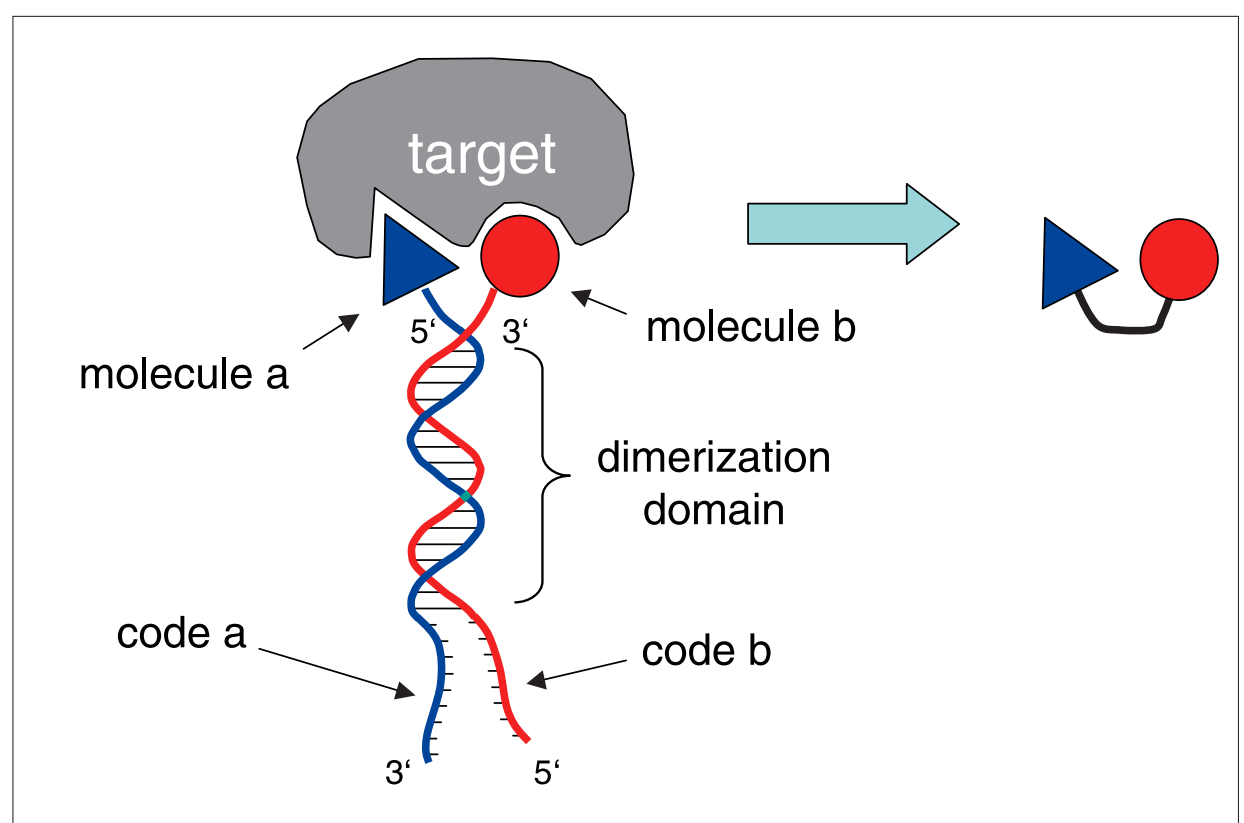

Fig. 5. Schematic representation of encoded self-assembling chemical libraries (ESACHEL) technology. Large libraries can be obtained by the self-assembly of smaller libraries of pharmacophores, covalently attached at the extremities of suitable oligonucleotides.

ganic molecule is linked to an individual oligonucleotide, which mediates the selfassembly of the library and provides a code associated to each binding moiety ('pharmacophore') (Fig. 5). The resulting library can be very large (as it originates from the combinatorial self-assembly of smaller sub-libraries). After the capture of the desired binding specificities on the target of interest, the 'binding code' can be 'decoded' by a number of experimental techniques (e.g. hybridization on DNA chips or by a modified polymerase chain reaction (PCR) technique followed by sequencing). Conveniently, at the end of the selection experiment the DNA is replaced by a suitable chemical linker, which connects the two synergistic pharmacophores (Fig. 5).

We have demonstrated the potential of ESACHEL technology in a recent article, in which we reported the construction of a first ESACHEL library and the isolation of binders to human serum albumin and carbonic anhydrase with affinities in the nanomolar range [14].

\section{Perspectives}

The targeted delivery of therapeutic agents to the tumor environment has yielded impressive results in aggressive animal models of cancer. Clinical trials are now urgently needed to shed light on the real potential of this approach to cancer therapy. Many antibody-based approaches to tumor targeting can be considered for therapeutic applications, immunocytokines being possibly the most straightforward class of bio- pharmaceuticals to characterize in clinical studies. In fact, several cytokines are now approved drugs, and the dose per patient is typically very low, thus simplifying GMP production of clinical-grade pharmaceuticals. The clinical applications of cytokines is often limited by unacceptably high toxicities, and their targeting to the tumor environment appears to be a logical step for improving the therapeutic ratio of these biopharmaceuticals. Only clinical trials will show whether antibody-cytokine fusions can be efficient and well-tolerated, or whether problems of immunogenicity, receptor trapping and insufficient targeting will limit the therapeutic performance in patients with cancer.

\section{Acknowledgements}

The tumor targeting research of the Neri group is financed by the ETH Zürich, the Swiss National Science Foundation (Projects 'Engineering and understanding the molecular targeting of angiogenesis' and 'TANDEM'), the Bundesamt für Bildung und Wissenshaft (EU Projects 'Therapeutic antibodies', 'FLUOR-MMPI' and 'STROMA'), the Gebert-Rüf Foundation and the Krebsforschung Schweiz.

Received: July 13, 2004

[1] L. Zardi, B. Carnemolla, A. Siri, T.E. Petersen, G. Paolella, G. Sebastio, F.E. Baralle, EMBO J. 1987, 6, 2337.

[2] B. Carnemolla, P. Castellani, M. Ponassi, L. Borsi, S. Urbini, G. Nicolo, A. Dorcaratto, G. Viale, G. Winter, D. Neri, L. Zardi, Am. J. Pathol. 1999, 154, 1345.

[3] D.M. Nanus, M.I. Milowsky, L. Kostakoglu, P.M. Smith-Jones, S. Vallaba- hajosula, S.J. Goldsmith, N.H. Bander, J. Urol. 2003, 170, S84.

[4] J. Rybak, S. Scheurer, D. Neri, G. Elia, Proteomics 2004, in the press.

[5] P. Oh, Y. Li, J., Yu, E. Durr, K.M. Krasinka, L.A. Carver, J.E. Testa, J.E. Schnitzer, Nature 2004, 429, 629.

[6] G. Winter, A.D. Griffiths, R.E. Hawkins, H.R. Hoogenboom, Annu. Rev. Immunol. 1994, 12, 433.

[7] F. Viti, F. Nilsson, S. Demartis, A. Huber, D. Neri, Methods Enzymol. 2000, 326, 480.

[8] P. Castellani, L. Borsi, B. Carnemolla, A. Biro, A. Dorcaratto, G. L. Viale, D. Neri, L. Zardi, Am. J. Pathol. 2002, 161, 1695.

[9] A. Pini, F. Viti, A. Santucci, B. Carnemolla, L. Zardi, P. Neri, D. Neri, J. Biol. Chem. 1998, 273, 21769.

[10] R. Fattorusso, M. Pellecchia, F. Viti, P. Neri, D. Neri, K. Wüthrich, Structure Fold. Des. 1999, 7, 381.

[11] F. Viti, L. Tarli, L. Giovannoni, L. Zardi, D. Neri, Cancer Res. 1999, 59, 347.

[12] L. Borsi, E. Balza, M. Bestagno, P. Castellani, B. Carnemolla, A. Biro, A. Leprini, J. Sepulveda, O. Burrone, D. Neri, L. Zardi, Int. J. Cancer 2002, 102, 75.

[13] M. Santimaria, G. Moscatelli, L. Giovannoni, A. Leprini, G. Neri, L. Zardi, D. Neri, P. Riva, Clin. Cancer Res. 2003, 9, 741.

[14] S. Melkko, J. Scheuermann, C. Dumelin, D. Neri, Nature Biotechnol. 2004, 22, 568. 